\title{
Image Analysis System for Detection of Red Cell Disorders Using Artificial Neural Networks
}

\author{
Ms. Y M Hirimutugoda BSc, MSc (IT) \\ Department of Computer Science and Engineering, Faculty of Engineering, University of Moratuwa, Moratuwa, \\ Sri Lanka \\ E-mail address: yasanthimalika@yahoo.com \\ Dr. Gamini Wijayarathna B.Sc, MEng, DrEng \\ Senior Lecturer, Department of Industrial Management, Faculty of Science, University of Kelaniya, Sri Lanka \\ E-mail address: gamini@kln.ac.lk
}

Sri Lanka Journal of Bio-Medical Informatics 2010;1(1):35-42

DOI: $10.4038 /$ sljbmi.v1i1.1484

\begin{abstract}
This paper investigates the possibility of rapid and accurate automated diagnosis of red blood cell disorders and describes a method to detect malarial parasites and thalassaemia in blood sample images acquired from light microscopes. As malaria and thalassaemia are life threatening diseases and an enormous global health problem, rapid and precise differentiation is necessary in clinical settings. The analysis of blood is a powerful diagnostic tool for the detection of these diseases. Visual inspection of microscopic images is the most widely used technique for determination of malaria and possible thalassaemia and it is a labour-intensive repetitive and time consuming task. Two back propagation Artificial Neural Network models (3 layers and 4 layers) was employed together with image analysis techniques to evaluate the accuracy of the classification in the recognition of medical image patterns associated with morphological features of erythrocytes in the blood. The three layers Artificial Neural Network (ANN) architecture had the best performance with an error of 2.74545e-005 and 86.54\% correct recognition rate. The trained three layer ANN acts as a final detection classifier to determine diseases. A medical consultation system has been jointly used with this system to provide clinical decision making ability. A questioning and answering dialog on the basis of patient history, physical examination and routine diagnostic test has been conducted in the medical consultation system with image analyzing result made by the trained ANN.
\end{abstract}

Keywords: Malaria; Thalassaemia; Artificial Neural Network; Erythrocytes

\section{Introduction}

Malaria, caused by a protozoan parasites of the genus Plasmodium, transmitted to people through the bites of infected mosquitoes, is the most serious and widespread parasitic disease of humans. A child dies of malaria every 30 seconds. The world malaria report 2008 describes that half of the world's population is at risk of malaria and an estimated 247 million cases led to nearly 881,000 deaths in $2006^{(1)}$. Of the four species known to infect humans, Plasmodium falciparum is the most virulent and contributes to the majority of deaths associated with the disease. But malaria is preventable and curable, if the patient is correctly diagnosed in early stage $^{(2)}$.

Thalassaemia is actually a group of inherited diseases of the blood that affect a person's ability to produce haemoglobin, resulting in anaemia. Haemoglobin is a protein in red blood cells that carries oxygen and nutrients to cells in the body. The World Health Organization (WHO) report of thalassaemia describes that each year about 300,000 infants worldwide are born with thalassaemia syndromes (30\%) or sickle-cell anaemia (70\%`). Globally, the percentage of carriers of Thalassaemia is greater than that of carriers of sickle-cell anaemia, but because of the higher frequency of the sickle-cell gene in certain regions, the number of affected births is higher than that of thalassaemia ${ }^{(3)}$. Thalassaemia occurs most frequently in people of Italian, Greek, Middle Eastern, Southern Asian and African Ancestry. 
Visual inspection of microscopic images is the most widely used technique for current determination of these blood disorders. Microscopy of Giemsa stained thick and thin blood films are used for the current standard determination of malaria. In peripheral blood sample, visual detection and recognition of Plasmodium spp is possible and effect via a chemical process called (Giemsa) staining. The staining process slightly colourises the red blood cells (RBCs) but highlights Plasmodium spp parasites, white blood cells (WBC), and platelets or artefacts. Detection of Plasmodium spp requires detection of stained objects. However, to prevent false diagnosis, the stained objects have to be analyzed further to determine if they are parasite or not ${ }^{(4)}$.

Although the microscopy has good sensitivity and allows species identification, there are some drawbacks. Visual inspection of microscopic images is time consuming and exhaustive. If the detection and counting process is interrupted, the operator has to start over again from the scratch. Different cells in microscope images can be differentiated by human visual analysis by using only the spatial and intensity information. After the blood cell slides have been analyzed, they are kept away. There is no quick and easy way of retrieving analyzing lot of images for future reference as with a computerized system. Some decision makers in emergency situations may not have accessed to test results before having to decide on treatment and they may have no experience of a particular rare condition and therefore not recognize it or not know how to deal with it. Emotional problems and fatigue degrade the expert's performance. Standardized automated image analysis software would circumvent limitations associated with manual determination ${ }^{(5)}$.

\section{Materials and Methods}

Artificial Neural Network (ANN) has been employed together with image processing techniques to automate the assessment of these blood disorders using the morphological features of erythrocytes in the blood. Prior to training, the first necessary step was to preprocess the Giemsa stained blood sample images acquired from using a high resolution digital camera mounted on a microscope. But the images were in various magnification factors, colour depths and images sizes. These acquired digital images were prone to a various types of noise which reduce the image quality. To prepare this data set for training and testing, common image processing tasks were performed on the digital images. Some of them are image enhancement, edge erosion, colour and size normalizing, specifying a region of interest, extraction of ROI, grey level conversion, reading pixel values.

\section{Image Processing}

A large number of various microscope images have to be collected for training and testing. According to the different magnification factors, the size of the red cell can change from an image to an image. To fix the average red cell size of the images into a standard size, the size normalization processed is done on the images according to magnification factors. The average diameter of the red cell was determined as 32 pixels according the evaluation performance of several different no of training cycles with the same dataset but the different average diameter red cell in the image. The accuracy is high when more information or a large number of pixels can be acquired from the blood cell.

It is essential to apply colour normalization to the images in order to decrease the effect of different light sources or sensor characteristics (e.g. intensity, white balance). Among many computational colour consistency algorithms based on the different models of illumination change, we have chosen to use an adapted grey world normalization method. According to 
this method it is assumed that the colour in each sensor channel averages to grey over the entire image. If it is not so, we wish to rotate the cluster to the main diagonal. Gray world normalization method based on the diagonal model of illumination change which utilizes certain characteristics of microscopic peripheral blood images. Gray level normalization assumes that there is a constant gray value of the image which does not change among different conditions ${ }^{(6)}$. In the diagonal model, an image of unknown illumination $\boldsymbol{I}^{u}$ can be simply transformed to the known illuminant space $\mathbf{I}^{k}$ by multiplying pixel values with a diagonal matrix ( $\left.\mathbf{I}^{\boldsymbol{k}}{ }_{\boldsymbol{r} \boldsymbol{g} \boldsymbol{b}(x)}(x) \mathbf{M I}^{\boldsymbol{u}}{ }_{r g b}(x)\right)$. Where $\mu^{\mathrm{I}}{ }_{r g b}$ are the means for channels $r, g, b$.

For ordinary images, normalization with a transformation using the average values yields poor results. However, the images subject to this study contain two basic components (plasma and the rest) which can be separated by a foreground and background segmentation. Hence, the grey value assumption can be successfully incorporated in to normalization process. In this method, the input image is first separated into foreground and background regions. According to the method described by $\mathrm{WHO}^{(3)}$ which use area morphology to estimate size of cells and then extracts foreground objects and estimates histograms. This procedure is quite efficient for the normalization of the image with respect to global illumination and staining effects.

It was decided to determine regions of interest of the larger captured images (some of which contain more than one suspicious area). The size of the ROI was originally chosen as it is large enough to accommodate the largest abnormal leukocyte with some headroom by inspecting images (300 images) randomly. A Region of Interest (ROI) was defined around the centre (cx, cy) of the suspicious area on the image. This region of interest was defined to be a square 160 by 160 pixels in size as minimum the training issues in the implementation stage.

A common pre-processing operation in image processing is the extraction of the region of the interest (ROI) which we wish to investigate more closely without the added complexity of extraneous data from other, unwanted, parts of an image. To do this we need to identify the region of interest and then crop, or cut away, this area from the rest of the image. The other reason for segmentation is to make sure that the ANN's is kept to the smallest possible size for in order to achieve easier training.

Due to edge effects in the captured images (the camera produced noticeable dark bands at the very edges of the image) there were many nuclear pixels around the perimeter of the image. An edge erosion filter, which simply set all the pixels within 3 pixels of the edge of the image to white, was developed and used.

\section{Training Neural Networks}

The pixel values of disease patterns on the suspected image areas have been input into two types of back propagation neural networks for training. Neural networks under investigation include (a) a three-layer and (b) a four-layer, fully connected, back-propagation architecture. There are 25,600 input neurons in the input layer $(25,600=160 * 160$ input image pixel matrix), 04 hidden neurons in the hidden layer(s), and four outputs in the output layer.

The numbers of the hidden neurons were chosen on the basis of trials with the training set to be as low as possible consistent with learning. Each pattern in the training and testing set consisted of a pattern name, $160 * 160$ pixel image data and a target output code. These have been shown in the table 1 . This illustrates the actual input and target output of cell patterns. 
Table 1. A sample dataset used for training

\begin{tabular}{|c|c|c|c|c|}
\hline & Malaria & Thalassemia & $\begin{array}{c}\text { Other } \\
\text { Abnormal }\end{array}$ & Normal \\
\hline $\begin{array}{l}\text { Blood image } \\
160 * 160 \text { pixels }\end{array}$ & "avgr & 年 & $\begin{array}{l}1.000 \\
0.80 \\
0.0\end{array}$ & 1000 \\
\hline Target output & 1000 & 0100 & 0001 & 0010 \\
\hline
\end{tabular}

The outputs from the neurons in the output layer of the neural network are presented in percentages, from $0 \%$ to $100 \%$.With respect to the target outputs, " 0 " equals $0 \%$ and " 1 " equals $100 \%$. The target outputs are defined as [1000] for Malaria, [0100] for Thalassaemia, [0001] for other abnormal and [0010] for normal. All the patterns were manually classified to define the target output. This ANN was trained with a standard back-propagation learning algorithm and weight was updated in batch mode. The network was trained for 1000 cycles. The number of training cycles was chosen after evaluating the performance of several different numbers of training cycles with the development set.

\section{Results of Two Neural Networks Architectures}

Three and four layer back-propagation ANNs were fully trained on the same image source. Performance was measured by the confusion matrix and data graph in each ANN. If any training errors and problems occurred, that was seen through a data graph. Figures 1 and 2 show the confusion matrix and the data graph of the 3 layers trained ANN. This data graph shows that this is a successful training and this confusion matrix shows that each disease has been correctly identified in training. The saved error of 3 layers and 4 layers ANN architectures were 2.74545e-005 and 2.82124e-005 respectively. Both ANNs were tested using the same image data set. The training data set was never used for testing. The 4 layer structure did not perform as well as the three layer one. Table 2 shows the results of the total correct recognition rate of all 2 ANN architectures. According to the results of minimum error rate and saved error, the 3 layers ANN was selected as a final classifier of this system. Further testing result of the 3 layer ANN with false negatives is shown in table 3.

Figure 1. Confusion matrix of trained 3 layers ANN

\begin{tabular}{|l|cccc|}
\hline \multicolumn{1}{|c}{ Nalaria } & Normal & 0ther & Thalassemia \\
\cline { 2 - 5 } Malaria & 100.000000 & 0.000000 & 0.000000 & 0.000000 \\
Iormal & 0.000000 & 100.000000 & 0.000000 & 0.000000 \\
Other & 0.000000 & 0.000000 & 100.000000 & 0.000000 \\
Thalassi & 0.000000 & 0.000000 & 0.000000 & 100.000000 \\
\cline { 2 - 4 } & & & & \\
\hline
\end{tabular}


Figure 2. Data graph of trained 3 layers ANN

-Active Cost

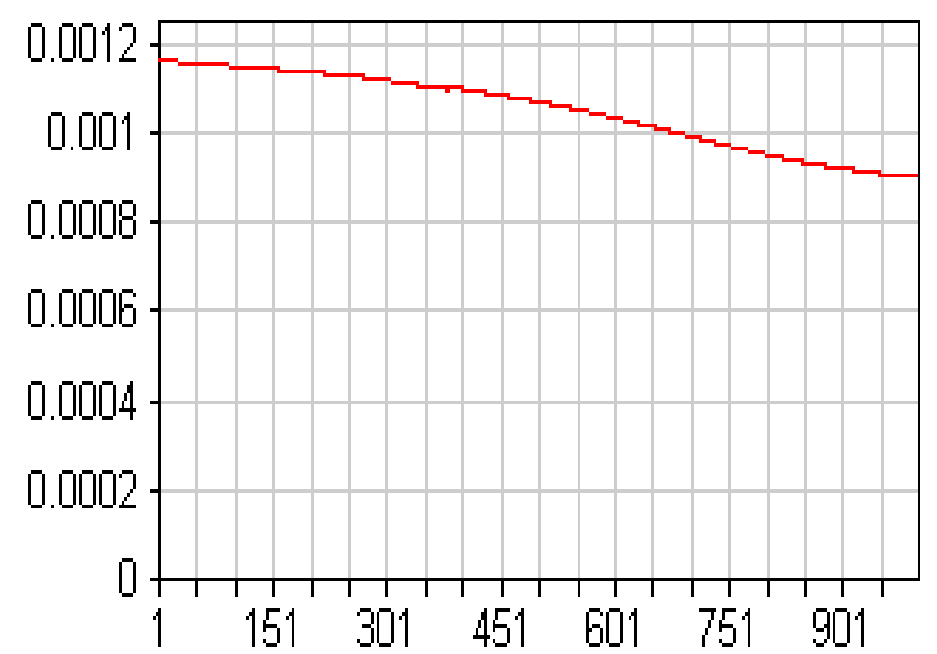

Table 2. Total correct recognition rates of both neural networks

\begin{tabular}{|c|c|l|l|l|}
\hline \multirow{2}{*}{ Number of Images } & \multirow{2}{*}{$\begin{array}{c}\text { Sample blood } \\
\text { Image type }\end{array}$} & \multicolumn{2}{c|}{$\begin{array}{c}\text { No of correct Recognition of } \\
\text { Testing }\end{array}$} \\
\cline { 4 - 5 } Training & \multirow{2}{*}{ Testing } & & $\begin{array}{c}\text { Three Layer } \\
\text { ANN }\end{array}$ & $\begin{array}{c}\text { Four } \\
\text { Layer } \text { ANN }\end{array}$ \\
\hline \multirow{2}{*}{120} & 70 & Malaria & $\begin{array}{l}61 / 70 \\
=87.14 \%\end{array}$ & $\begin{array}{l}56 / 60 \\
=80.00 \%\end{array}$ \\
\hline \multirow{2}{*}{120} & \multirow{2}{*}{80} & Thalassaemia & $\begin{array}{l}72 / 80 \\
=90.00 \%\end{array}$ & $\begin{array}{l}67 / 70 \\
=83.75 \%\end{array}$ \\
\hline \multirow{2}{*}{120} & \multirow{2}{*}{60} & Normal & $\begin{array}{l}52 / 60 \\
=85.00 \%\end{array}$ & $\begin{array}{l}50 / 60 \\
=83.33 \%\end{array}$ \\
\hline \multirow{2}{*}{120} & \multirow{2}{*}{50} & Other abnormal & $\begin{array}{l}42 / 50 \\
=84.00 \%\end{array}$ & $\begin{array}{l}40 / 50 \\
=80.00 \%\end{array}$ \\
\hline \multicolumn{2}{|l|}{ The total average correct recognition rate } & $86.54 \%$ & $84.25 \%$ \\
\hline \multicolumn{2}{|l|}{ Saved error } & & $2.74545 \mathrm{e}-005$ & $2.82124 \mathrm{e}-005$ \\
\hline
\end{tabular}

Table 3. Number of incorrect recognition images of 3 layer ANN

\begin{tabular}{|l|l|l|l|l|l|}
\hline \multirow{2}{*}{ Image Type } & \multicolumn{5}{|c|}{ No of Incorrect Recognitions of Testing 3 Layer ANN } \\
\cline { 2 - 7 } & Total & Malaria & Thalassemia & Normal & Abnormal \\
\hline Malaria & $9 / 70$ & - & 3 & 2 & 4 \\
\hline Thalassemia & $8 / 80$ & 2 & - & 1 & 5 \\
\hline Normal & $8 / 60$ & 2 & 2 & - & 4 \\
\hline Other abnormal & $8 / 50$ & 4 & 3 & 1 & - \\
\hline Total no of false negatives $20 / 260=7.6 \%$ & & & \\
\hline
\end{tabular}




\section{Medical Consultation System}

The system is in this mode when a user is interacting with it. The dialog structure is the user interface that allows the user to interact with system to obtain results and query the system. This was mainly indicated when considering possible malaria types on the basis of patient history, physical examination, and routine diagnostic tests. Information representation procedure in this medical consultation system was developed in the form of "IF....THEN structure" rules. The following illustrates how typical rules run from medical consultation system with the user response.

Rule $p_{1}$

\begin{tabular}{|c|c|}
\hline $\begin{array}{l}\text { IF } \\
\text { AND }\end{array}$ & $\begin{array}{l}\text { poor general health } \\
\text { temperature }>=38^{\circ} \mathrm{C}\end{array}$ \\
\hline AND & enlarged spleen \\
\hline THEN & treatment for Malaria \\
\hline
\end{tabular}

Rule $p_{3}$

$\begin{array}{ll}\text { IF } & \text { fever } \\ \text { AND } & \text { sweating } \\ \text { AND } & \text { chills }\end{array}$

THEN $\quad$ poor general health

Patient Facts

$\begin{array}{ll}f_{5}: & \text { fever } \\ f_{6}: & \text { sweating } \\ & \ldots \ldots \ldots \ldots \ldots \\ f_{n}: & \text { chills } \\ f m: & \text { temperature }>=38^{\circ} \mathrm{C}\end{array}$

\section{Discussion and Conclusion}

The classification accuracy of 86.54\% with 3 layers ANN was achieved in this study. The goal was largely met and false negative rates (7.6 \%) were extremely low. The false negatives that were found were mostly in blood cell images with very brightness ranges or less contrast than other cell images. This initial study is promising and shows that direct classification with image pixels intensity data through ANN is possible, even some back ground variation in the image.

Neural networks are generally perceived as being a 'black box'. It is extremely difficult to document how specific classification decisions are reached. In a medical decision support system, asking questions, giving explanations and reasons and dealing with uncertainties are very important things. As a solution for these important issues, an Expert System was created. But interfaces problems were encountered when combining the Expert System with this trained Neural Network to give the reasoning and explanation power to this system.

We are also aiming at a possible change to the algorithms to find a better approach for the highest accuracy of this solution. Currently I am studying to apply following research based approaches to overcome the above problems and to achieve $100 \%$ or a comparable higher 
accuracy for this solution.

The study of why and how the misclassification occurred is very necessary and important. Previous studies also have cases that were misclassified; however, it is uncommon to see the explanations for the above misclassification. The analysis of misclassification result has been identified as a future research area that may achieve a clinical application potential. According to this research area, in the current study we used the Support Vector Machine (SVM) classifier to find the better accuracy classifier for this medical problem using the same medical dataset. If the merits of the Support Vector Machines and Artificial Neural Networks can be combined, we can obtain a new Artificial Neural Network Support Vector Machine with a better performance. Motivated by this idea, we hope to apply a recurrent Neural Network to SVM training for this medical image pattern recognition. Within this research area, we are also studying how to use the Multi Agents System for training this medical image dataset to get the result and find the accuracy of using a Multi Agents System.

The use of hypermerge hybrid systems can be used to address problems in computer-assisted decision making. Hybrid systems use combined methodologies from two or more techniques in the same system. Often, these include the combination of knowledge-based methods with data-based methods such as neural networks. In addition, various techniques are used to handle uncertain information, including fuzzy set theory, approximate reasoning. In my current study, the structural components of them are examined to know how to these methods are integrated.

\section{Acknowledgements}

We would like to thank Dr K A C Wickramarathne Consultant Hematalogist / Senior Lecturer in Pathology, Department of Pathology, Faculty of Medicine, University of Ruhuna and to Dr Renuka and Ms Pathma in Karapitiya Teaching Hospital, Galle.

\section{References}

1. World Health Organization. What is Malaria? http://apps.who.int/gb/ebwha/pdf_files/EB118/B118_5-en.pdf.

2. Dacremont V, Landry P, Mueller I, Pecoud A. Clinical and laboratory predictors of imported Malaria in an outpatient setting: An aid to medical decision making in returning travelers with fever. The American Society of Tropical Medicine and Hygiene 2002;481-6.

3. World Health Organization. What is Thalassaema? http://apps.who.int/gb/ebwha/pdf_files/EB118/B118_5-en.pdf.

4. BorayTek F, Dempster AG, Kale I. Malaria parasite detection in peripheral blood images. British Machine Vision Conference, 2006;347-56.

5. Szolovits P, Patil RS, Schwartz WB. Artificial intelligence in medical applications. Annals of International Medicine 1988;108:80-8.

6. Ross NE, Pritchard CJ, Rubin DM, Duse AG. Automated image processing method for the diagnosis and classification of Malaria in thin blood smears. Medical and Biomedical Engineering and Computing 2006;44:427-36.

7. Gonzalez RC, Woods RE. Digital Image Processing. 
8. Zahir S, Chowdhury R, Payne GW. Automated Assessment of Erythrocyte Disorders Using Artificial Neural Network. IEEE International Symposium on Signal Processing and Information Technology 2006;776-80.

9. Zheng Q, Milthorpe BK, Jones AS. Direct Neural Network Application for Automated Cell Recognition. Cytometry Part A: The Journal of the International Society for Analytical Cytology 2004;57:1-9.

10. Sheikh H, Bin Zhu, Micheli-Tzanakou, E. Blood cell identification using neural networks. Bioengineering Conference, In proceedings of the 1996 IEEE TwentySecond Annual Northeast, 1996;119-20

11. Kim KS, Kim PK. Analyzing Blood Cell Images to distinguish its Abnormalities”, $8^{\text {th }}$ ACM international conference on Multimedia 2000;395-7.

12. Hudson DL, Cohen ME, Networks and Artificial Intelligence for Biomedical Engineering. Wiley-Blackwell. 2000.

13. Tan Y, Xia Y, Wang J. Neural network realization of support vector methods for pattern classification. Proceedings of the IEEE-INNS-ENNS International Joint Conference 2000;6:411-16.

14. Ramana KV, Khader Basha S. Neural image recognition system with application to tuberculosis detection. Proceedings. ITCC 2004. International Conference on Information Technology: Coding and Computing 2004;2:694-98.

15. Chen P, Yan X, Zheng C. Recognition of Blood and Bone Marrow Cells using Kernelbased Image Retrieval. IJCSNS International Journal of Computer Science and Network Security 2006; 6:29-34. 